\title{
CARLOS GATTI MURRIEL
}

UN TESTIMONIO AGRADECIDO

\section{Jorge Wiesse Rebagliati* \\ Universidad del Pacifico \\ wiesse_jr@up.edu.pe}

Resumen: El presente ensayo plantea la importancia de la experiencia docente, en el testimonio de vida de Carlos Gatti Murriel, como guía para la formación integral de los jóvenes universitarios y para el soporte de una

* Jorge Wiesse Rebagliati es licenciado en Lingüística y Literatura por la Pontificia Universidad Católica del Perú. Ha sido vicedecano de Estudios Comunes y pertenece a la Asociación Internacional de Hispanistas, a la Sociedad Peruana de Estudios Clásicos, al Centro de Investigación de la Universidad del Pacífico y al Instituto RivaAgüero de la Pontificia Universidad Católica del Perú. Actualmente, se desempeña como profesor principal del Departamento Académico de Humanidades de la Universidad del Pacífico y codirector del boletín Coiné de esta casa de estudios. Sus poemas, artículos y contribuciones han aparecido en revistas especializadas y diarios locales. Ha publicado el poemario Vigilia de los sentidos (Laberintos, 2005), el volumen colectivo La divina comedia. Voces y ecos (Lima: Universidad del Pacífico, 2008) y Otros textos. Apropiaciones, 1989- 2009 (Lima: Universidad del Pacífico, 2010). En el 2014, tradujo y realizó el posfacio de Fernando Pessoa. 35 Sonnets/35 sonetos (Lima: Trashumantes, 2014) y editó junto a Jerónimo Pizarro, Los futuros de Fernando Pessoa (Lima: Universidad del Pacífico, 2014). En 2015 editó el libro colectivo Purgatorios. Purgatori (Lima: Universidad del Pacífico e Instituto Italiano de Cultura). 
vida académica a la que debe aspirar toda universidad. Para tal efecto, se consideran dos factores determinantes y trascendentes que caracterizan la vida de Carlos Gatti Murriel. Por un lado, su sapiencia producto de los años de estudio, de su encuentro con los Clásicos, y de la convivencia con grandes maestros de las letras; y, por otro lado, su calidad humana — reflejada en su capacidad de acogimiento - la cual ha sido fundamental y contundente para asumir la ardua tarea de todo educador: humanizar al hombre.

Primeramente, se recoge la riqueza de su enseñanza en la Facultad de Letras de la Católica y en el Instituto Riva-Agüero, para cuyos alumnos el encuentro con Gatti significó la experiencia raigal de lo que era una universidad. Luego, se valora su vinculación con la música, sus dones musicales los aplica tanto en la docencia como en funciones académicas relevantes. En tercer lugar, se resalta su fecunda experiencia en la Universidad del Pacífico hasta la actualidad. Asimismo, se valora su generosa entrega para con otras casas de estudios; así como la experiencia de la "Lectura Dantis" como parte del camino de humanización del hombre. Finalmente, se concluye argumentando que su trabajo debe bastar para dar por buena, por bella y por verdadera la opción universitaria de toda una vida.

Palabras Clave: Carlos Gatti Murriel, Instituto Riva-Agüero, tutoría, música, docencia, literatura italiana, Dante.

\section{CARLOS GATTI MURRIEL}

\section{A grateful testimony}

ABSTRACT: The present test raises the importance of the educational experience, in the testimony of Carlos Gatti Murriel's life, as guide for the integral formation of the university young persons and for the support of an academic life to the one that must inhale any university. For such an effect, they are 
considered to be two determinant and transcendent factors that characterize Carlos Gatti Murriel's life. On the one hand, his wisdom product of the years of study, of his meeting with the Classic ones, and of the conviviality with big teachers of the letters; and, on the other hand, his quality humanizes - reflected in his capacity of welcome — which has been fundamental and forceful to assume the arduous task of every educator: To humanize the man.

Firstly, retires the wealth of his education in the Faculty of Letters of the Catholic one and in the Institute Riva-Agüero, for whose pupils the meeting with Gatti meant the experience raigal of what was a university. Then, his entail is valued by the music, applies his musical gifts both in the teaching and in academic relevant functions. Thirdly, his fecund experience is highlighted in the University of the Pacífico up to the current importance. Likewise, his generous delivery is valued for by other universities; as well as the experience of the "Lectura Dantis" as part of the way of humanization of the man. Finally, there ends up by being argued that his work must be enough to give for good, for beautiful and for real the university option of the whole life. KeYwords: Carlos Gatti Murriel, Institute Riva-Agüero, tutorship, music, teaching, Italian literature, Dante.

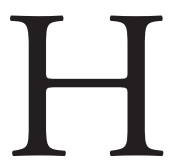

ay una breve narración de Borges — "Del rigor en la ciencia”, en El Hacedor - en la que el autor argentino imagina un mapa que coincide con la realidad representada. Luego de un tiempo indeterminado, el mapa se rompe en pedazos. En los pedazos habitan animales y mendigos.

Cuando lo conocí, en el invierno de 1971, Carlos Gatti podía hacer algo análogo con el tiempo: Carlos podía reproducir con precisión absoluta aquello que había hecho en la misma fecha del año anterior. Idealmente, podía 
reproducir todo el día del año anterior, con lo que cabía la posibilidad de que un día de su vida fuera la repetición cabal de uno previo. Esto, por supuesto, es una fantasía: la narración siempre condensa, siempre resume, lo que contamos no coincide al ciento por ciento con lo que vivimos; pero ello no impide el asombro y la maravilla.

Aún hoy, en sus juveniles 70, Carlos luce una memoria prodigiosa, pues su erudición absolutamente natural nunca es acumulativa: siempre surge de una innata búsqueda de orden. Probablemente esta cualidad, unida a una curiosidad voraz, cause la impresión de que siempre estemos conversando no con un mero interesado, sino con un especialista. $Y$ sus intereses son amplísimos. Yo oí — serían los años 80 - a Alberto Escobar, famoso lingüista y profesor sanmarquino, referirse a Carlos como "un Leonardo".

En efecto, Carlos no solamente maneja con amplitud y solvencia temas literarios y musicales (digámoslo así: su “core-business"), sino también una amplia gama de temas científicos. Sabe de Anatomía, de Botánica (¿quién de ustedes sabe, por ejemplo, que el nombre científico del molle, de donde se extrae la llamada "pimienta del Perú" es "schinus molle"?: quizás Charo Gómez, Carlos Amat y León o Pacho Roca, pero no muchos más), de Física, de Meteorología, de Astronomía; no es mal matemático (como lo hace nuestro amigo común, el profesor Ricardo Siu, Carlos cuenta por grupos y en bulto, lo que prueba su innata intuición numérica). Es, con seguridad, un geógrafo notable.

Una vez, mientras él cuidaba un examen (lo que prueba que el multitasking no es una actividad absolutamente reciente) se entretuvo mentalmente, enumerando las ciudades que conocía de Lombardía, la región italiana. Contó 78. Y estaban ordenadas, de norte a sur y de este a oeste. Puede, ciertamente, reproducir también con exactitud la toponimia menor de la ruta Chancay-Casma, para angustioso deleite de sus sobrinos. 
Con todo lo dicho, probablemente, lo que me impresionó más fue su enorme capacidad de acogimiento. No dudo de que sea este rasgo de su personalidad el que le ha permitido alcanzar en varios años la distinción de profesor mejor evaluado del Departamento Académico de Humanidades de la Universidad del Pacífico. A veces, cuando lo observo actuar en clase o en otras reuniones académicas siempre me asombran tanto su paciencia como su capacidad para extraer de una observación o una pregunta a veces extravagante, a veces digresiva, todo un potencial significativo que empata con naturalidad con el tema central.

Sin esta voluntad de acogimiento, no puede entenderse cómo él, a sus sólidos 28 años — diez de los cuales había dedicado ya a la docencia—, poseedor de un mítico prestigio académico, se ocupara de las inquietudes del muchacho de 17 años lleno de lecturas dispersas, un poco desorientado, bastante perplejo, que era yo en ese entonces. Ni que dedicara sus horas libres de los sábados o los domingos (luego de una semana empeñadísima, pues trabajaba en el Instituto Riva-Agüero, en la Universidad de Lima y en la Facultad de Letras de la Católica) a formarnos en gramática, en métrica y en el estudio de la lírica española contemporánea — me refiero al grupo de alumnos de Estudios Generales Letras que él convocó y en el que estaban también Alonso Cueto, María Gracia Martínez y Rosa Samamé-, y luego, a ver películas en el cine club del Ministerio de Trabajo o del Touring Club.

Eran días que terminaban alrededor de una mesa en la que también — con toda naturalidad y sin presiones de ningún tipo- comentábamos libros y películas en medio de observaciones relativas a los sucesos del día. Con otros compañeros — veo en este momento al Negro Vega y a Miguel Giusti en el primer patio de Letras en la Plaza Francia y también a Milena Cáceres y a Edgar O'Hara - el entusiasmo no era menor, aunque la frecuencia no fuera la misma. En casa de Carlos, en el centro de Lima, con otros amigos 
(recuerdo a mi hermano Ricardo, a Alejo Ferreyros, a Aldo Gatti, a Úrsula Ramírez, a los Carvallo - Constantino, Fernando y Carmen-y a Carlos García, entre otros; a Eduardo Lores, a Alberto Benavides y a Charo Saco los conocí después) oíamos música (la clásica, ciertamente, pero también la popular). Podría ser que esta cualidad, el acogimiento, fuera parte del ADN familiar: la simpatía de don Carlos Gatti Brunialti rompía inmediatamente cualquier distancia y los Murriel, la familia de la señora Amelia, la madre de Carlos, por tradición secular, estaban acostumbrados a recibir en la casa hacienda de Jaihua, en el valle medio del río Casma, a los viajeros importantes que hacían la ruta Casma-Huaraz o viceversa; dońa Amelia recibía con gentileza y cuidado en su casa, y todos sus hijos - María Teresa y María del Carmen, Aldo, Rafo y Gina — la han seguido en esto, pero en Carlos el acogimiento es de una evidencia que impacta.

En retrospectiva, y luego de haber conocido la realidad de universidades anglosajonas y europeas, siento que la Católica de los años 70, o más específicamente, Riva-Agüero, y aún más específicamente, la presencia amical y docente de Carlos, resultó para nosotros la experiencia raigal de lo que era una universidad. Aprendíamos conversando con el Dr. De la Puente, con Armando Nieto o con Margarita Guerra; aprendíamos oyendo discutir en el segundo patio de la casona Riva-Agüero a Ricardo González Vigil y a Manuel Migone sobre Joyce y Proust; a César Gutiérrez, un ayacuchano de solera, formulando sus dudas acerca de la posibilidad de un mundo andino moderno o antihispánico. Aprendíamos con un seminario que aparentemente no tenía que ver con lo inmediato o con lo pragmáticamente aplicativo.

Porque, ¿̇de qué podía servirle a un muchacho de 18 años leer a san Ireneo de Lyon, un padre de la Iglesia del siglo II d.C., guiado por Mons. Óscar Alzamora (quien se había especializado en Patrística en el Seminario de los Marianistas en Friburgo)? Pero le sirvió. Y presidiendo todo este proceso, 
la orientación benéfica de Carlos, quien bebió este estilo universitario en el Seminario de Filología del Instituto Riva-Agüero y en la Facultad de Letras de la Católica, con maestros como Luis Jaime Cisneros, discípulo a su vez de Amado Alonso, o como Gred Ibscher, alumna dilecta de Werner Jaeger (o como Purificación Estébanez, Enrique Carrión y Armando Zubizarreta), y con compañeros entrañables — se aprende también, y mucho, de los compañeros - como Alberto Hernández, Rosa Luisa Rubio, José Urrutia y José Luis Rivarola. Libertad académica, ósmosis de la excelencia y tutoría amical: es una fórmula imbatible. Que lo diga George Steiner.

En Escrito a ciegas, el poeta Martín Adán dice: "Si quieres saber de mi vida, vete a mirar al Mar". Carlos Gatti podría decir: "Si quieres saber de mi vida, vete a oír a Schumann”. O a Mozart. O a Schubert. O a Liszt. En suma: vete a oír música clásica (aunque también la popular: Carlos conoce con detalle de verdadero experto la canción italiana de los años 60 , gusta de los huainos y alguna vez intentó estudiar arpa andina: el intento quedó en eso, pues el profesor se asustó cuando Carlos le dijo que sabía leer música).

Resulta imposible no referirse a la música a propósito de Carlos Gatti. Esto debido a que no solo es un buen pianista formado en la Academia Moderna de Piano dirigida por María Ureta del Solar, alumna del gran pianista chileno Claudio Arrau, y un buen musicólogo (ha escrito un libro sobre Franz Liszt), alumno de personalidades tan relevantes como Rodolfo Holzmann, Hans Günther Mohmer y Albor Maruenda, sino porque Carlos piensa, digámoslo así, musicalmente. Ello le permite, por ejemplo, seguir varias conversaciones a la vez, como si el conjunto fuera una fuga de Bach, a varias voces.

La utilidad extramusical de este don la descubrió Carlos Gatti cuando se desempeñó, durante 21 años y un poc mas, como Secretario General de la Universidad del Pacífico. En esta función debía seguir simultáneamente, 
varias conversaciones, en las sesiones del Consejo Universitario o en las reuniones de la Asamblea Universitaria. Su vinculación de años (superan los 45) con la Sociedad Filarmónica de Lima es fruto del convencimiento de que la experiencia de la música debe viralizarse, porque algo tan bello y tan pleno no puede — no debe- quedarse solamente en uno.

A esta orientación hacia lo sonoro debe Carlos su interés por la Fonética y por la Métrica. Por años, Carlos dictó clases de Fonética General y de Fonética y Fonología del Español en la Universidad Católica. A los alumnos de Letras se les hacía cuesta arriba tratar de manejar conceptos acústicos que exigían un mínimo de Matemáticas (logaritmos, por ejemplo). Sin embargo, lo más difícil venía después: teniendo en cuenta la transcripción fonética con caracteres del Alfabeto Fonético Internacional, los alumnos debían memorizar un texto breve y pronunciarlo a la perfección.

En la época de exámenes finales, el patio de la vieja casa Riva-Agüero, en el centro histórico de Lima, donde Carlos los tomaba, se volvía una Babel en miniatura. En el estilo de la escena final de Farenheit 451, la película de François aे Truffaut inspirada en la novela de Ray Bradbury, donde — para preservar la cultura de los libros de un estado totalitario que los quemaba mediante bomberos incendiarios - cada lector se había memorizado un libro (cada lector era un libro). En el patio de Riva-Agüero, cada alumno de Fonética recitaba la fábula de Esopo del viento norte y el sol en un idioma distinto: húngaro, ruso, japonés, polaco, sueco, vietnamita, turco...

Su vínculo con la Universidad del Pacífico se retrae a la amistad con Pedro Benvenutto, quien fue rector de esta casa de estudios entre 1971 y 1977. Recuerdo que, luego del terremoto de 1974, Carlos y yo acompañamos a Pedro a revisar los daños que este había causado en la Universidad. A diferencia de lo que vemos hoy, no tuvimos que caminar mucho: la planta física de la Universidad estaba compuesta por la antigua casona — que se encontraba donde 
ahora está el local de la Biblioteca—, el pabellón B, el auditorio Maes-Heller y la canchita. La cafetería (que estaba donde está el jardín contiguo a la canchita) y el Centro de Investigación —una hilera de oficinas donde ahora se encuentra el pabellón A - se habían construido con materiales provisionales. El Centro de Investigación estaba sobre dos tiendas - me parece que eran una bodega y una farmacia - que la Universidad alquilaba y que daban a la Av. Sánchez Cerro.

Cuando Pedro Benvenutto murió en 1978 —velaron su cuerpo en la Universidad: los testimonios de afecto y dolor, sobre todo de los alumnos, fueron conmovedores - , las autoridades del momento, Estuardo Marrou, el Padre Villagrasa y José Javier Pérez, nos llamaron a Carlos Gatti y a mí para que nos ocupáramos de dictar los cursos de Lenguaje que él dejó. Para nosotros fue un reto: tanto por la responsabilidad que suponía estar a la altura de un gran profesor - y un gran lingüista — como Pedro Benvenutto, como porque nosotros veníamos de la Católica (Carlos dictaba también Lenguaje y Literatura en la de Lima), y estábamos acostumbrados a un público que acogía sin resistencias mayores los temas humanísticos. Decidimos aprovechar partes del curso de Pedro Benvenutto, pero, básicamente, imaginamos uno nuevo, adecuado a las necesidades del alumno de la Universidad del Pacífico. Como no encontramos materiales que nos fueran útiles para ello, los creamos, usando libremente el método comunicativo y una versión ecléctica de la gramática funcional. Con modificaciones importantes, los materiales se convirtieron en libros con que se formaron generaciones de alumnos de la Universidad del Pacífico y también de otras universidades. En aquellos tiempos, tuvimos la ayuda de destacados alumnos de la Universidad, que nos apoyaron como jefes de prácticas, como Víctor Flores, Ana María Montenegro, Vilma Yep, Meche Aráoz y Magali Silva, y de jóvenes profesores, como Javier Bustillos y José Antonio Rodríguez. Eleonora Falco, Cecilia Lesevič, Martina Vinatea, Pepe Güich, Lucho Vargas y Paco Tumi se incorporaron poco tiempo después. 
Creo que Carlos Gatti le dio a la Universidad del Pacífico la claridad y la serenidad de su consejo a lo largo de los 21 ańos y nueve meses en que ejerció de Secretario General. Durante ese periodo siempre dictó por lo menos un curso: Lenguaje III o Literatura Universal. Y en su ejercicio docente y en el de gobierno procuró proyectar con lealtad el ideal vitalmente académico de la universidad que vivió en la Católica de los 60 y que yo pude gozar en el Instituto Riva-Agüero de los 70. No estuvo solo en esto ni fue una tradición espuria en la Pacífico, que ha contado siempre - no solo ahora - con un poderoso Departamento de Humanidades, como lo puede certificar la presencia seminal de figuras como las del P. Enrique López Dóriga S.J., recientemente fallecido, o Ángel Palencia S.J., César Pacheco Vélez y Percy Cayo, entre otros. Dentro de estos “otros”, agrego al grupo al Padre Alfredo Montemayor S.J., al "Che" Montemayor, porque — en verdad - no hay tanta diferencia entre los departamentos de Ciencias Sociales y de Humanidades, y el Che fue un humanista cabal y un gran amigo. Si hubiera sido un vino, habría sido un vino humanista D.O.C.: de denominación de origen controlada.

Carlos fue el profesor fundador del curso de Literatura Italiana en la Facultad de Letras y Ciencias Humanas de la Universidad Católica. Y en los 43 años que siguieron a aquel lejano 1972, su único profesor. Todos los primeros semestres de esos años, los nombres de Cavalcanti, Dante, Petrarca, Ungaretti, Svevo, Tomasi di Lampedusa, Buzzati y muchos más se volvieron vida en la interioridad de alumnos receptivos y asombrados. Sus contribuciones a las universidades a las que ha ofrecido su dedicación generosa (la Universidad Femenina del Sagrado Corazón, la Universidad de Lima, la Universidad Católica Sedes Sapientiae y la Universidad Antonio Ruiz de Montoya) han suscitado siempre la gratitud de alumnos y autoridades. Recientemente, la Universidad del Pacífico lo distinguió con el 
Profesorado Emérito y la Universidad Ruiz de Montoya, con el Profesorado Emérito Extraordinario y la medalla “Juan Pablo Viscardo y Guzmán”.

Fuera de la estructura curricular, y quizás surgido de la superabundancia del curso de Literatura Italiana, probablemente el empeño más relevante de Carlos Gatti sea la lectura de las obras de Dante Alighieri, la Lectura Dantis, las reuniones de lectura semanales de lectura de la Divina Comedia o de la Vida nueva en el original italiano, y luego en traducción al español (hecha por Carlos), que se enriquece con comentarios relativos al texto que siempre termina en una interpretación de lo contemporáneo realizada por un grupo plural que incluye a personas - alumnos, profesores, profesionales, entusiastas todos — dedicadas a distintas actividades (en algún momento coincidieron Sergio Bazán, Quique Espinoza, Marisol Palacios, Harold Hernández, Camilo Torres y David Wong, por ejemplo: un abogadoeditor, dos antropólogos, una directora de obras de teatro, un escritor y un especialista en finanzas; actualmente, un economista, Mario Aguirre, convoca y anima nuestras reuniones).

Además de los artículos puntuales que Carlos le ha dedicado a la obra de Dante y que continúan una tradición dantesca peruana que viene desde el siglo XVI (el primer testimonio es de un encomendero toledano que escribe en el Cuzco), sigue con textos del XVII y continúa con Corpancho en el XIX, y con varios estudiosos en el XX, entre ellos Julio Picasso y Leopoldo Chiappo, junto con todo esto, Carlos Gatti ha traducido la Divina Comedia al español, una traducción de trabajo que esconde con pudor poco frecuente en otros.

La Lectura Dantis empezó como empezaron las universidades en el Medioevo: varios alumnos se acercaron a Carlos para pedirle que dirigiera un grupo de lectura de la Divina Comedia. Recuerdo que en el grupo inicial estaban Eduardo Chirinos y Mario Ghibellini, y algunos hinchas más. Y se 
inició en el Instituto Riva-Agüero en 1984. Continuó en la Universidad del Pacífico, donde nos reunimos desde 1988. Y se mantiene aquí hasta ahora, fecundando desarrollos creativos de distinto tipo, como los objetos plásticos de Luis Alfredo Agusti, Ricardo Wiesse o Susan Zimič, los poemas de Giovanna Pollarolo o Elio Vélez o míos, o los artículos y las investigaciones de Martina Vinatea y otros.

Personalmente, vuelvo siempre a los problemas que Carlos suscitó en mí en el curso de Literatura I, a los temas que juntos desarrollamos a lo largo de años de colaboración, a las lecturas y a los autores que él me sugirió, a él, para una consulta puntual o un consejo. Y Dante — como la música, Roma o el paisaje del norte peruano- es para nosotros siempre tierra común y fuente de prodigios. Creo que la continuidad de una obra tan fecunda en las obras y en el mundo interior de sus alumnos, que inevitablemente se transforman en sus amigos, debería bastar para dar por buena, por bella y por verdadera la opción universitaria de toda una vida. 